\title{
Combining Uncertainty and Vagueness in Time Intervals
}

\author{
Christophe Billiet and Guy De Tré \\ Department of Telecommunications and Information Processing, Ghent University, \\ Sint-Pietersnieuwstraat 41, B-9000, Ghent, Belgium \\ Christophe.Billiet@UGent.be, Guy.DeTre@UGent.be
}

\begin{abstract}
Database systems contain data representing properties of real-life objects or concepts. Many of these data represent time indications and such time indications are often subject to imperfections. Although several existing proposals deal with the modeling of uncertainty or vagueness in time indications in database systems, only a few of them summarily examine the interpretation and semantics of such imperfections. The work presented in this paper starts at a more thorough examination of the semantics and modeling of uncertainty or vagueness in time intervals in database systems and presents methods to model combinations of uncertainty and vagueness in time intervals in database systems, based on examinations of their requisite interpretations.
\end{abstract}

\section{Introduction}

Information systems in general and database systems (DBS) in specific usually have two main purposes: to preserve data, information or knowledge concerning real-life objects or concepts and to allow humans to retrieve these data, information or knowledge. In order to do this, DBS usually dispose of data representing properties of these real-life objects or concepts. Through such data (a.o.), DBS model these real-life objects or concepts [2], [14]. For example, patient DBS may model patients' medical state by containing data representing these patients' heart rate, blood pressure, ...

Many real-life objects or concepts are somehow time-related. Thus, DBS often dispose of data representing time indications [1], [11], [15], [17]. Time indications modeled by DBS refer to parts of time and generally take the form of either time intervals [3], [10] or instants [3], [10]. A time interval can intuitively be seen as a continuous period of time, whereas an instant can intuitively be seen as an infinitesimally short 'moment' in time [3], [10]. For example, patient DBS may need to model patients' medical history by containing data representing the time intervals during which the patients suffered from certain diseases or data representing the instants when the patients took medication. Because time intervals in DBS are studied more exhaustively in literature [1], [2], [5], [8], [12], [14], [15], [16], [17] than instants in DBS [7], [8] and because an instant can be seen (and modeled) as a time interval, the work presented in this paper only considers time intervals in DBS. 
Usually, a lot of the data disposed of by DBS are created by humans or represent information or knowledge created by humans. Such data represent properties which can be the result of measurements, estimations, derivations, ... However, human-made data, information and knowledge can be prone to imperfections, due to the inherent imperfect nature of humans and human reasoning or imperfections in measuring equipment [1], [2], [5], [6], [7], [8], [9], [11], [12], [13], [14], [15]. For example, a medical professional may need to estimate the exact moment in time when a patient was given a certain medicine, as nobody remembers exactly, or the period of time during which a patient suffered from a certain disease, fully knowning that the emergence and the disappearance of the disease are gradual. Obviously, the degree to which data, information or knowledge preserved in an DBS present a correct reflection of reality and thus are any useful, is heavily influenced by the degree to which the imperfections in these data, information or knowledge can be defined and described in the DBS. For example, if a patient DBS only allows to specify one single exact date on which a patient started suffering from a disease, medical professionals will never be able to make the information modeled by this DBS reflect the gradual emergence and disappearance of a disease over the period of several days. Thus, the authors of this paper strongly believe that modeling imperfections in data, information or knowledge to incorporate such imperfections into DBS is highly preferable to only allowing perfect, but inherently wrong data, information or knowledge into DBS. Hence, the work presented in this paper considers modeling time intervals subject to imperfection in DBS.

The two most common types of imperfections to which time intervals in DBS could be subject, are [2], [5], [8], [11], [12], [14], [15], [16], [17]:

- Uncertainty. A time interval is subject to uncertainty when it is known that a precise time interval is intended, but it is somehow uncertain which interval this is. For example, surgery on a patient may have started exactly at $7 \mathrm{~h} 42$ and ended exactly at $11 \mathrm{~h} 46$, but a secretary, not knowning this, might incorporate in the patient DBS that surgery started 'around 8' and ended 'around 12'. Exact, precise starting and ending instants exist, but the secretary doesn't know them.

- Vagueness/imprecision. A time interval is subject to imprecision or vagueness when it is certainly known exactly which time interval is intended, but not every instant belongs to this time interval to the same extent. For example: it could be known for sure that a patient showed no symptoms of a disease up to $8 \mathrm{~h} 12$ and all the possible symptoms of this disease starting from 10h11. In this case, the patient is considered ill with this disease starting at $8 \mathrm{~h} 12$, but in the period between $8 \mathrm{~h} 12$ and $10 \mathrm{~h} 11$, the patient isn't considered to be fully sick yet. At every instant between $8 \mathrm{~h} 12$ and 10h11, he is only ill with the disease to some degree. When such imprecision is described or indicated using linguistic terms, it is called vagueness.

Up to now, many proposals have considered the modeling of uncertainty [1], $[2],[4],[15],[14]$ or vagueness [17] in time intervals modeled by DBS. Surprisingly, 
to the knowledge of the authors, only very few proposals examine the interpretation and semantics of imperfections in time intervals modeled by DBS and all proposals doing this, do it in a very summary way. Even more surprisingly, to the knowledge of the authors, no proposal has ever considered the modeling of combinations of both uncertainty and vagueness in time intervals modeled by DBS. However, the authors deem it likely that situations exist where it is necessary to be able to model such combinations. For example: different sources may disagree about the time interval subject to vagueness during which a patient became increasingly ill with a disease, resulting in uncertainty about which time interval is the correct one.

In order to respond to such situations, the work presented in this paper makes three contributions:

1. Both the modeling and semantics of uncertainty and vagueness in time intervals modeled by DBS are clearly explained. This is done in section 3 .

2. The semantics of possible combinations of uncertainty and vagueness in time intervals modeled by DBS are explored and based on this exploration, a technique to model these combinations will be presented. This is done in section 4 .

3. It is the opinion of the authors that (at least) one combination could semantically be reduced to a more usable form. The main arguments for this opinion and a proposed reduction strategy will be introduced in section 5 .

First, however, section 2 presents and describes a few necessary concepts.

\section{Preliminaries}

\subsection{Time As Perceived by Database Systems}

Time is usually perceived by DBS as a linear given that flows at a constant pace and in one direction (from the past to the future). Therefore, time is often thought of in the context of DBS as following an axis (a time axis), where the points on this axis are infinitesimally short 'moments' in time [2], [8], [11], [12], [14], [15], [17]. Thus, time is often seen as a totally ordered set of such points (the time axis), which are called instants [3], [10].

Definition 1. Instant [3], [10]

An instant is a time point on an underlying time axis.

For example, the point on an unspecified time axis described by the words 'March 6 th at $19 \mathrm{~h}$ exactly' is an instant.

Using two of its instants, an interval subset of a time axis can be defined. Such interval subset is called a time interval [3], [10].

Definition 2. Time interval [3], [10]

In the presented work, a subset of all instants of a time axis between two given instants of this time axis is called a time interval 
For example, the subset of an unspecified time axis described by the words 'from March 6th at 19h exactly until March 7th at noon exactly' is a time interval, bounded by the instants described by the words 'March 6th at $19 \mathrm{~h}$ exactly' and 'March 7th at noon exactly'.

In theory, time intervals can be closed, halfopen or open intervals. An instant itself is in fact a closed time interval of which the bounding instants coincide. The work presented in this paper deals with imperfection in closed time intervals.

Definition 3. Duration [3], [10]

$A$ duration is an amount of time with known length, but no specific starting or ending instants.

A time interval is bounded by two instants, whereas a duration is not. For example, the amount of time described by the words 'a month' is a duration: its length is given, but its bounding instants are not.

\subsection{Time As Modeled by Database Systems}

As many properties of real-life objects or concepts are time-related, many DBS model time intervals or instants using time models. However, as DBS are usually finitely precise and instants have an infinitesimally short duration, time models used by DBS usually model an underlying time axis using chronons.

Definition 4. Chronon [3], [10]

In a data model, a chronon is a non-decomposable time interval of some fixed, minimal duration.

A time model used by an DBS usually disposes of a datatype used to represent time indications. Such datatype usually has a time domain at it's disposal, which is a set of values allowed by the datatype. For example, a time model's datatype could use $\mathbb{Z}$ as time domain.

Usually, time models used by DBS model a time axis using a sequence of consecutive chronons. Every element of the time model's time domain then corresponds to exactly one chronon, the ordering of the consecutive time domain elements reflecting the temporal ordering of these consecutive chronons. These chronons are the smallest time intervals an DBS using the time model can distinguish, which is generally why they are used [11], [15].

An instant is usually modeled by a time model as a single element of a time domain, corresponding to the single chronon containing the instant somehow. A time interval can be modeled as an interval in the time domain, corresponding to a set of consecutive chronons called a chronon interval.

Definition 5. Chronon interval

In a data model, a chronon interval is a set of (one or more) consecutive chronons, used to represent a time interval. 
An example is in order here. Consider an DBS with a time model modeling a time axis, where this time axis is a totally ordered set containing all instants in the first week of the year 2014. The time domain $\mathbb{E}$ used by this model could for example be the interval $\mathbb{E}=[1,7] \subset \mathbb{N}$. Every different element $e$ of $\mathbb{E}$ could now correspond to a different chronon $c$, which have a duration of one day. For example: element 1 corresponds to the first day of 2014, element 2 to the second, etc. A (closed) time interval starting half an hour before the start of the second day of 2014 and ending two hours before the end of the second day of 2014 can now be mapped to a chronon interval consisting of only the chronons corresponding to the first and second day of 2014, and thus be represented by time domain interval $[1,2]$.

The work presented in this paper deals with imperfection in both closed time intervals and closed intervals in time domains (which are called closed time domain intervals in this paper). Approaches to determine chronons or chronon intervals or elements of or intervals in a time domain based on corresponding instants or time intervals are not dealth with in this paper.

\section{Uncertainty and Vagueness in Time (Domain) Intervals}

In sections 3.1 and 3.2, the semantics and interpretations of respectively uncertainty and vagueness in time (domain) intervals are clearly explained and the ways to model these are described. For reasons of clarity, an interval which is not subject to any imperfections will be called a regular interval in this paper.

\subsection{Uncertainty in Time (Domain) Intervals: Semantics and Modeling}

Although other sources may exist, uncertainty in time indications modeled by DBS is usually caused by a (partial) lack of knowledge: the exact part of time intended by the time indication exists, but uncertainty exists concerning exactly which part of time is intended, because knowledge about the intended part of time is incomplete. This is mainly because DBS model time concerning real-life objects or concepts, and in most cases, temporal aspects of real-life objects or concepts can simply not be perfectly and completely controlled. For example, a medieval document may be written on one specific day in history, but it might be unknown exactly which day this was, resulting in uncertainty about the exact day intended.

Therefore, the work presented in this paper only considers time (domain) intervals subject to uncertainty caused by a (partial) lack of knowledge. Confidence in the context of such uncertainty should be modeled using possibility theory [1], [2], [4], [7], [8], [9], [15]. In the work presented in this paper, possibility is always interpreted as plausibility, given all available knowledge.

The work presented in this paper will introduce the modeling of uncertainty in time (domain) intervals using the concept of ill-known intervals (IKI). However, before introducing IKI, the concepts of possibilistic variables and ill-known values (IKV) should be introduced [1], [2], [4], [14], [15]: 


\section{Definition 6. Possibilistic variable}

$A$ possibilistic variable $X$ on a universe $U$ is a variable taking exactly one value in $U$, but for which this value is (partially) unknown. The possibility distribution $\pi_{X}$ on $U$ models the available knowledge about the value that $X$ takes: for each $u \in U, \pi_{X}(u)$ represents the possibility that $X$ takes the value $u$.

Consider a universe $U$ and a possibilistic variable $X$ on $U$, defined and described by its possibility distribution $\pi_{X}$ on $U$. For every $u \in U, \pi_{X}(u)$ now represents the possibility that $X$ takes the value $u$. The interpretation is that $\pi_{X}(u)$ represents how plausible it is that $X$ takes the value $u$, given all available knowledge about the value $X$ is intended to take, fully understanding that it is (partially) unknown which value $X$ is intended to take.

\section{Definition 7. Ill-known value}

Consider a set $U$ containing single values (and not collections of values). When a possibilistic variable $X_{\tilde{v}}$ is defined on $U$, the unique value $X_{\tilde{v}}$ takes, which is (partially) unknown, will be a single value in $U$ and is called an illknown value (in $U$ ).

Consider a set $U$ of single values and an IKV $\tilde{v}$ in $U$ defined and described by a possibilistic variable $X_{\tilde{v}}$ on $U$, which is defined and described by its possibility distribution $\pi_{X_{\tilde{v}}}$ on $U$. The IKV $\tilde{v}$ now intends to be a single value in $U$. However, due to a (partial) lack of knowledge about the exact value $\tilde{v}$ is intended to be, it is not certain exactly which value $\tilde{v}$ is. The degree of possibility $\pi_{X_{\tilde{v}}}(u)$ of an arbitrary element $u$ of $U$ is now interpreted as the degree of plausibility that $X_{\tilde{v}}$ takes value $u$ and thus that $\tilde{v}$ is $u$, given all available knowledge about the value $\tilde{v}$ is intended to be, fully understanding that it is (partially) unknown which value $\tilde{v}$ is intended to be.

\section{Definition 8. Ill-known interval}

Consider a totally ordered set $U$ containing single values (and not collections of values) and its powerset $\wp(U)$. Now consider the subset $\wp_{I}(U)$ of $\wp(U)$ and let this subset contain every element of $\wp(U)$ that is an interval, but no other elements. When a possibilistic variable $X_{\tilde{I}}$ is defined on this $\wp_{I}(U)$, the unique value $X_{\tilde{I}}$ takes, which is (partially) unknown, will be a regular interval in $U$ and is called an ill-known interval (in $U$ ).

Consider a totally ordered set $U$ of single values and an IKI $\tilde{I}$ in $U$ defined and described by a possibilistic variable $X_{\tilde{I}}$ on $U$, which is defined and described by its possibility distribution $\pi_{X_{\tilde{I}}}$ on $U$. The IKI $\tilde{I}$ now intends to be a single regular interval in $U$. However, due to a (partial) lack of knowledge about the exact interval $\tilde{I}$ is intended to be, it is uncertain exactly which regular interval $\tilde{I}$ is. The degree of possibility $\pi_{X_{\tilde{I}}}(J)$ of an arbitrary regular interval $J \subseteq U$ is now interpreted as the degree of plausibility that $X_{\tilde{I}}$ takes value $J \in \wp_{I}(U)$ and thus that $\tilde{I}$ is $J$, given all available knowledge about the regular interval $\tilde{I}$ is intended to be, fully understanding that it is (partially) unknown which value $\tilde{I}$ is intended to be. 
In the work presented in this paper, an IKI in a time axis is called an Ill-known Time Interval (IKTI), while an IKI in a time domain is called an Ill-known Time Domain Interval (IKTDI).

In conclusion, it should be clear that the occurrence of uncertainty in time (domain) intervals only implies that the time (domain) interval intended is not exactly known, as some of the knowledge to determine this is lacking. The occurrence of uncertainty in time (domain) intervals does not imply that the intended time (domain) interval isn't a precise one.

\subsection{Vagueness in Time (Domain) Intervals: Semantics and Modeling}

As mentioned before, many time intervals modeled in DBS correspond to real-life objects or concepts, which could be (to some degree) defined by humans. Often, such objects or concepts may have a gradual nature: at some instants, they are in existence to a higher extent than at other instants. At some instants, they are fully non-existing, at others they are fully in existence and at yet others, they only exist to some (and not full) extent. For example, the 'industrial revolution' is a concept defined by humans based on the developmental state of a part of the world. It took place during the 18th and 19th century, so one could correspond it to the time interval containing every instant between the first instant of the 18th century and the last instant of the 19th century. However, as the developmental state of the world gradually changed (and the Industrial Revolution thus gradually came into existence) between 1750 and 1800 and the inverse took place during the middle of the 19th century, the instants belonging to these parts of time belong to the time interval corresponding to the industrial revolution to a lesser extent than the instants belonging to the part of time between 1800 and the middle of the 19th century, when the industrial revolution was in full existence.

The time intervals corresponding to such 'gradual' objects or concepts contain every instant which corresponds to the object or concept to some degree. However, some instants fully are moments in time when the object or concept is in existence and thus fully belong to its corresponding time interval and some instants merely partially correspond to moments in time when the object or concept is in existence and thus merely partially belong to its corresponding time interval.

As a result of the gradualness of such object or concept, (parts of) the time interval corresponding to it can only be approximately described. When such a description is given using precise boundaries, the time interval is subject to imprecision. For example: the industrial revolution gradually came into existence between 1750 and 1800. Thus, a part of the time interval during which the industrial revolution was in existence is approximately described using the precise boundaries 'between 1750 and 1800'. When such a description is given using linguistic terminology, the time interval is subject to vagueness. For example: the industrial revolution gradually faded out during the middle of the 19th century. Thus, a part of the time interval during which the industrial revolution was in 
existence is approximately described using the linguistic description 'the middle of the 19th century'.

The work presented in this paper will introduce the modeling of imprecision or vagueness in time (domain) intervals using the concept of fuzzy time (domain) intervals $(\mathrm{FT}(\mathrm{D}) \mathrm{I})$.

\section{Definition 9. Fuzzy Time Interval}

Consider a time axis $T$, which is a totally ordered set of instants. A fuzzy time interval $\tilde{V}$ (in $T$ ) is a fuzzy subset of $T$ of which the support is an interval in $T$ and which has a clearly conjunctive interpretation.

\section{Definition 10. Fuzzy Time Domain Interval}

Consider a time domain $D$, which is a totally ordered set. A fuzzy time domain interval $\tilde{W}$ (in $D$ ) is a fuzzy subset of $D$ of which the support is an interval in $D$ and which has a clearly conjunctive interpretation.

The imprecision or vagueness in a time (domain) interval corresponding to a real-life object or concept can now be modeled by allowing the time (domain) interval to be a FT(D)I. Given a time axis $T$ and a FTI $\tilde{V}$ in $T$ defined by its membership function $\mu_{\tilde{V}}$, the membership degree $\mu_{\tilde{V}}(u)$ of instant $u \in T$ now expresses to what extent $u$ belongs to $\tilde{V}$. Such a membership degree can be interpreted in two ways.

- Similarity. Here, instants at which the real-life object or concept fully exist are given the highest possible membership degrees. The membership degree of any other instant then quantifies the extent to which that instant is similar to the aforementioned instants, where two instants are more similar when their extents to which the real-life object or concept exists at them are more similar.

- Truth or preference. Here, an FTI corresponding to a real-life object or concept is seen as a set of all instants at which the real-life object or concept existed. The membership degree of an instant is seen as reflecting the extent to which the person(s) that defined the FTI find(s) it true or preferred that the real-life object or concept exists at this instant.

In conclusion, it should be clear that the occurrence of imprecision or vagueness in time (domain) intervals only implies that not every instant belongs to the time (domain) interval to the same degree. However, it is perfectly and certainly known which instants belong to the time (domain) interval to which degree.

\section{Combining Uncertainty and Vagueness in Time (Domain) Intervals}

In this section, two types of situations are presented, in which it appears necessary to model combinations of uncertainty and vagueness in time (domain) intervals. Both situation types are accompanied by explanatory examples. For every situation type, a technique for modeling the imperfection in the time (domain) intervals is presented. 


\subsection{Uncertainty About a Time (Domain) Interval Subject to Vagueness: Semantics and Modeling}

In this type of situation, it is perfectly known that a real-life object or concept corresponds to a single time (domain) interval subject to vagueness, however, it is (partially) unknown exactly which time (domain) interval subject to which vagueness this is. As a result, there exists uncertainty concerning which time (domain) interval subject to vagueness corresponds to the object or concept.

Consider a situation in which both a nurse and a doctor examine a patient, searching for the evolution of a disease in the patient. The doctor finds no signs of the disease in the patient up to instant $t_{d, 1}$, all signs of full disease starting from instant $t_{d, 2}$ and ending at instant $t_{d, 3}$, and no signs anymore starting from instant $t_{d, 4}$, where $t_{d, 1}$ is earlier than $t_{d, 2}$, which is earlier than $t_{d, 3}$, which is earlier than $t_{d, 4}$. The doctor concludes that the time interval during which the patient is ill is the interval starting at $t_{d, 1}$ and ending at $t_{d, 4}$ and that the parts of the time interval between $t_{d, 1}$ and $t_{d, 2}$, resp. $t_{d, 3}$ and $t_{d, 4}$ are subject to imprecision. The nurse now finds the same general disease evolution, but concludes that the time interval during which the patient is ill is the interval starting at $t_{n, 1}$ and ending at $t_{n, 4}$ and that the parts of the time interval between $t_{n, 1}$ and $t_{n, 2}$, resp. $t_{n, 3}$ and $t_{n, 4}$ are subject to imprecision, where $t_{n, 1}$ is earlier than $t_{n, 2}$, which is earlier than $t_{n, 3}$, which is earlier than $t_{n, 4}$. Thus, there is uncertainty about the time interval during which the patient is ill, which is a time interval subject to imprecision.

In the work presented in this paper, it is suggested to model all imperfection in such types of situations by making the time (domain) interval under consideration an ill-known FT(D)I (IKFT(D)I).

\section{Definition 11. Ill-known Fuzzy Time Interval}

Consider a time axis $T$, which is a totally ordered set of instants. Consider the fuzzy powerset $\tilde{\wp}(T)$ of $T$ and let this set contain every fuzzy subset of $T$ and no other elements. Now consider the subset $\tilde{\wp}_{\tilde{V}}(T)$ of $\tilde{\wp}(T)$ and let this set contain every fuzzy time interval in $T$ and no other elements. When a possibilistic variable $X_{\tilde{\tilde{V}}}$ is defined on this $\tilde{\rho}_{\tilde{V}}(T)$, the unique value $X_{\tilde{\tilde{V}}}$ takes, which is (partially) unknown, will be a fuzzy time interval in $T$ and is called an ill-known fuzzy time interval (in $T$ ).

\section{Definition 12. Ill-known Fuzzy Time Domain Interval}

Consider a time domain D, which is a totally ordered set. Consider the fuzzy powerset $\tilde{\wp}(D)$ of $D$ and let this set contain every fuzzy subset of $D$ and no other elements. Now consider the subset $\tilde{\wp}_{\tilde{V}}(D)$ of $\tilde{\wp}(D)$ and let this set contain every fuzzy time domain interval in $D$ and no other elements. When a possibilistic variable $X_{\tilde{V}}$ is defined on this $\tilde{\wp}_{\tilde{V}}(D)$, the unique value $X_{\tilde{\tilde{V}}}$ takes, which is (partially) unknown, will be a fuzzy time domain interval in $T$ and is called an ill-known fuzzy time domain interval (in $D$ ).

An IKFT(D)I now intends to be an FT(D)I, thus, a time (domain) interval subject to vagueness or imprecision. This is modeled through the use of 
the set of every existing FT(D)I in the definition of the IKFT(D)I, where the FT(D)I inherently model vagueness or imprecision. However, due to a (partial) lack of knowledge, there exists uncertainty about exactly which FT(D)I is the one intended by the IKFT(D)I. Confidence in the context of this uncertainty is modeled using the possibilistic variable on the set of existing FT(D)I.

\subsection{Vagueness About Time (Domain) Intervals Subject to Uncertainty: Semantics and Modeling}

In this type of situation, there is no intention to represent a single time (domain) interval. Instead, the intention is to represent a set of time (domain) intervals, all of which may belong to this set to different degrees. However, as a result of a (partial) lack of knowledge, it is not known exactly which time (domain) interval is intended by every element of the aforementioned set. As a result, in this type of situation, the intention is to represent a set of time (domain) intervals, for some of which there exists uncertainty about exactly which time (domain) interval is intended.

Consider a hospital where a secretary attempts to model the surgery scheduling preferences of a surgeon. Consider the surgeon telling her he can't perform surgery before 10 o'clock because of a meeting ending at 10, which could run late up to 15 minutes. After the meeting, he could perform surgery, but only mildly prefers to do so. From around 12 o'clock until around 13 o'clock (15 minutes of delay could occur) is his lunch break and after that, he prefers most to perform surgery. Thus, the surgeon has given the secretary two intervals in time during which he prefers (more or less) to perform surgery, but due to possible delay of other obligations, it is not known exactly which time intervals are intended.

In the work presented in this paper, it is suggested to model all imperfection in such types of situations by making the time (domain) interval under consideration a fuzzy $\operatorname{IKT}(D) I(\mathrm{FIKT}(\mathrm{D}) \mathrm{I})$.

\section{Definition 13. Fuzzy Ill-known Interval}

Consider a totally ordered set $U$ containing single values (and not collections of values). Now consider the subset $\tilde{\wp}_{\tilde{I}}(U)$ of $U$ and let this set contain every ill-known interval in $U$, but no other elements. A fuzzy ill-known interval (in $U$ ) is a fuzzy subset of $\tilde{\wp}_{\tilde{I}}(U)$ which has a clearly conjunctive interpretation.

A fuzzy ill-known interval (FIKI) in a time axis is now called a Fuzzy Illknown Time Interval (FIKTI), whereas a FIKI in a time domain is called a Fuzzy Ill-known Time Domain Interval (FIKTDI).

A FIKT(D)I now models a set of time (domain) intervals, where uncertainty about exactly which time (domain) intervals are intended is modeled through the use of IKT(D)I. However, different IKT(D)I may have different membership degrees in the set, representing the different extent to which they belong to the set, modeling imprecision or vagueness about this set of IKT(D)I. Such membership degrees can now again be interpreted as degrees of similarity or degrees of truth or preference. 


\section{A Reducible Combination}

Consider a type of situation where one wants to model uncertainty about a time (domain) interval subject to uncertainty. In theory, it would be possible to model this as an IKV in a set of IKI in a time axis or time domain. Semantically, this construction intends to be an IKT(D)I, however, there is uncertainty about exactly which IKT(D)I is intended. Moreover, every IKT(D)I intends to be a regular time (domain) interval, however, there is uncertainty about exactly which interval is intended. Overall, this construction intends to be a regular time (domain) interval, however, there is uncertainty about exactly which interval is intended. Thus, it is the opinion of the authors that situations of this type could be modeled using a single IKT(D)I.

Consider a totally ordered set $U$ containing single values (and not collections of values). Now consider the subset $\tilde{\wp}_{\tilde{I}}(U)$ of $U$ and let this set contain every IKI in $U$, but no other elements. Now consider an $\operatorname{IKV} \tilde{\tilde{I}}$ in $\tilde{\wp}_{\tilde{I}}(U)$ defined by the possibility distribution $\pi_{X_{\tilde{I}}}$ of its possibilistic variable. Now consider an arbitrary element $\tilde{I}_{i}$ of $\tilde{\wp}_{\tilde{I}}(U)$, defined by the possibility distribution $\pi_{X_{\tilde{I}_{i}}}$ of its possibilistic variable. For an arbitrary regular interval $I$ in $U, \pi_{X_{\tilde{I}_{i}}}(I)$ now expresses the possibility that $I$ is the regular interval intended by $\tilde{I}_{i}$, whereas $\pi_{X_{\tilde{I}}}\left(\tilde{I}_{i}\right)$ expresses the possibility that the interval intended by $\tilde{\tilde{I}}$ is the interval intended by $\tilde{I}_{i}$. Following the rules of possibility theory [7], the possibility $\pi(I)$ that the interval intended by $\tilde{\tilde{I}}$ is the interval $I$ should thus be calculated as follows.

$$
\pi(I)=\max _{\tilde{I}_{i} \in \tilde{\S}_{\tilde{I}}(U)}\left(\min \left(\pi_{\tilde{I}_{i}}(I), \pi_{\tilde{\tilde{I}}}\left(\tilde{I}_{i}\right)\right)\right)
$$

Thus, a construction as introduced above can semantically be reduced to a single IKI.

\section{Conclusions and Future Work}

In the work presented in this paper, the semantics and interpretation of uncertainty and vagueness in time (domain) intervals in database systems are thoroughly examined. Based on the semantics and interpretations of combinations of such uncertainty and vagueness, methods to model such combinations are introduced. A reducible combination is identified and described. Future work should either focus on the remaining combination or on the temporal relationships between such combinations and regular time intervals. As stressed throughout the paper, interpretation and semantics should never be downplayed.

\section{References}

1. Billiet, C., Pons Frias, J.E., Pons, O., De Tré, G.: Bipolarity in the Querying of Temporal Databases. In: Atanassov, K.T., Homenda, W., Hryniewicz, O., 
Kacprzyk, J., Krawczak, M., Nahorski, Z., Szmidt, E., Zadrozny, S. (eds.) New trends in fuzzy sets, intuitionistic fuzzy sets, generalized nets and related topics volume II: applications, pp. 21-37. SRI PAS/IBS PAN, new trends edn. (2013)

2. Billiet, C., Pons Frias, J.E., Pons Capote, O., De Tré, G.: A Comparison of Approaches to Model Uncertainty in Time Intervals. In: Pasi, G., Montero, J., Ciucci, D. (eds.) Advances in Intelligent Systems Research. pp. 626-633. Atlantis Press, Milano, Italy (2013)

3. Böhlen, M.H., et al.: The Consensus Glossary of Temporal Database ConceptsFebruary 1998 Version. Lecture Notes in Computer Science 1399/1998, 367-405 (1998)

4. Bronselaer, A., Pons, J.E., De Tré, G., Pons, O.: Possibilistic evaluation of sets. International Journal of Uncertainty, Fuzziness and Knowledge-Based Systems 21(3), 325-346 (2013)

5. Chountas, P., Petrounias, I.: Modelling and Representation of Uncertain Temporal Information. Requirements Engineering 5(3), 144-156 (2000)

6. De Tré, G., Zadrozny, S., Bronselaer, A.J.: Handling Bipolarity in Elementary Queries to Possibilistic Databases. IEEE Transactions on Fuzzy Systems 18(3), 599-612 (2010)

7. Dubois, D., Hadjali, A., Prade, H.: A Possibility Theory-Based Approach to the Handling of Uncertain Relations between Temporal Points. International Journal of Intelligent Systems 22(2), 157-179 (2007)

8. Dubois, D., Prade, H.: Processing Fuzzy Temporal Knowledge. IEEE Transactions on Systems, Man and Cybernetics 19(4), 729-744 (1989)

9. Dubois, D., Prade, H.: The Three Semantics of Fuzzy Sets. Fuzzy Sets and Systems 90(2), 141-150 (1997)

10. Dyreson, C.E., et al.: A Consensus Glossary of Temporal Database Concepts. SIGMOD Record 23(1), 52-64 (1994)

11. Dyreson, C.E., Snodgrass, R.T.: Supporting Valid-Time Indeterminacy. ACM Transactions on Database Systems 23(1), 1-57 (1998)

12. Garrido, C., Marín, N., Pons, O.: Fuzzy Intervals To Represent Fuzzy Valid Time in a Temporal Relational Database. International Journal of Uncertainty, Fuzziness and Knowledge-Based Systems 17(Suppl .1), 173-192 (2009)

13. Kacprzyk, J., Zadrozny, S.: Computing with Words in Intelligent Database Querying: Standalone and Internet-based Applications. Information Sciences 134(1-4), 71-109 (2001)

14. Pons, J.E., Marín, N., Pons, O., Billiet, C., Tré, G.D.: A Relational Model for the Possibilistic Valid-time Approach. International Journal of Computational Intelligence Systems 5(6), 1068-1088 (2012)

15. Pons Frias, J.E., Billiet, C., Pons, O., De Tré, G.: Aspects of Dealing with Imperfect Data in Temporal Databases. In: Pivert, O., Zadrozny, S. (eds.) Flexible Approaches in Data, Information and Knowledge Management, chap. 9, pp. 189220. Springer, flexible a edn. (2013)

16. Qiang, Y., Asmussen, K., Delafontaine, M., Stichelbaut, B., De Tré, G., De Maeyer, P., Van De Weghe, N.: Handling Imperfect Time Intervals in a Two-Dimensional Space. Control and Cybernetics 39(4), 983-1010 (2010)

17. Schockaert, S., Cock, M.D.: Temporal reasoning about fuzzy intervals. Artificial Intelligence 172, 1158-1193 (2008) 